\title{
Universal principles governing multiple random searchers on complex networks: The logarithmic growth pattern and the harmonic law
}

\author{
Tongfeng Weng, ${ }^{1,2, *}$ Jie Zhang, ${ }^{3}$ Michael Small, ${ }^{4,5}$ Bahareh Harandizadeh, ${ }^{2}$ and Pan Hui ${ }^{2}{ }^{\dagger}$ \\ ${ }^{1}$ Business School, University of Shanghai for \\ Science and Technology, Shanghai 200093, China \\ ${ }^{2}$ HKUST-DT System and Media Laboratory, \\ Hong Kong University of Science and Technology, HongKong \\ ${ }^{3}$ Centre for Computational Systems Biology, Fudan University, China \\ ${ }^{4}$ The University of Western Australia, Crawley, WA 6009, Australia \\ ${ }^{5}$ Mineral Resources, CSIRO, Kensington, WA, Australia
}

(Dated: March 5, 2018)

\begin{abstract}
We propose a unified framework to evaluate and quantify the search time of multiple random searchers traversing independently and concurrently on complex networks. We find that the intriguing behaviors of multiple random searchers are governed by two basic principles - the logarithmic growth pattern and the harmonic law. Specifically, the logarithmic growth pattern characterizes how the search time increases with the number of targets, while the harmonic law explores how the search time of multiple random searchers taken with respect to that needed by individual searchers. Numerical and theoretical results demonstrate these two universal principles established across a broad range of random search processes, including generic random walks, maximal entropy random walks, intermittent strategies, and persistent random walks. Our results reveal two fundamental principles governing the search time of multiple random searchers, which are expected to facilitate investigation of diverse dynamical processes like synchronization and spreading.
\end{abstract}

PACS numbers: 89.75.Hc, 05.40.Fb, 05.60.Cd

*Electronic address: wtongfeng2006@163.com

${ }^{\dagger}$ Electronic address: panhui@cse.ust.hk 
It has long been recognized that diverse dynamics of natural and artificial systems ranging from the stochastic motion of molecules [1], animals foraging [2], transportation [3], to information and disease spreading [4] can be described well and accurately modeled by random search processes on a network. The broad range of relevances have turned random search processes on complex networks into a long-standing topic of scientific interest [5-8]. Amongst random search processes, it is increasingly recognized that often more than one target was need to be found or captured in the task of each individual search, for example, cells chasing pathogens [9], predators harvesting prey [10], and even the project of boosting search methods [11]. For characterizing multiple targets search, a growing number of studies are based on the quantity search efficiency function that describes the ratio of the number of targets visited to the total distance traversed by the searcher [12-16]. Here, we consider another appealing quantity mean random cover time (MRCT) - the expected time required for the discovery or exploration of a number of targets given in advance. Moreover, a substantial body of research has shown that mean random cover time has profound implications, generating random spanning trees in computer science [17] and solving the well-known coupon collector's problem [18].

Unfortunately, the studies on mean random cover time has achieved little progress. Most previous work either pays attention to this problem on regular graphs or obtain some numerical findings [19-21]. Important steps were achieved in Ref. [11], where the random cover time of various search strategies was found to follow a universal distribution resembling the Gumbel distribution. Interestingly, this finding seems to imply a logarithmic growth pattern between the search time and the number of targets, which is further recovered and extended in our recently work [22]. Notably, all these findings were so far limited to a single searcher. Several searchers moving and traveling concurrently for target search, however, is commonly encountered in real life, for example, a prey species surviving around several distinct predators. Previous studies have demonstrated that multiple random searchers are faster than one in the case of the exhaustive search [23, 24].

In this paper, we address the general problem of finding a number of targets by multiple random searchers on networks and provide a theoretical framework to determine mean random cover time analytically. Interestingly, we find two fundamental principles (i.e., the logarithmic growth pattern and the harmonic law) controlling search time of multiple random searchers for finding multiple targets, given in advance. In particular, the logarithmic 
growth pattern describes the effects of the number of targets, while the harmonic law explores the effects and contributions of the individual searchers. These two universal principles are confirmed across a broad range of the stochastic processes, including generic random walks [5], maximal entropy random walks [25], persistent random walks [26], and intermittent search strategies [27]. Our work provides a general framework for studying random search processes taking place on networks.

We start from $K$ identical random searchers moving concurrently and independently on a network. For simplicity, here we assume that there is no information sharing among these searchers during the entire search process. At each time step, the individual searcher jumps from current node $i$ to node $j$ with the transition probability $p_{i j}$, which is a time-invariant quantity. In this context, an interesting problem is how long do these searchers take to find multiple targets given in advance on the network? We denote by $T_{V_{i}, \Omega_{m}}^{(K)}$, defined as the expected time needed to visit $m$ targets located at the node set $\Omega_{m}$ starting from the location $V_{i}$ for the $K$ searchers. Note that once a given target is found by any random searcher, it will disappear in the target set and also become invisible to other searchers. To derive this interesting quantity analytically, we first consider a single target case for which $\Omega_{m}=\{j\}$ and $V_{i}=\left\{v_{1}, v_{2}, \cdots, v_{K}\right\}$. In this situation, if the first step of any searcher is to node $j$, the expected number of steps required is 1 ; if all of them are to some other nodes $V_{i}^{\prime}=\left\{v_{i}^{\prime} \mid v_{i}^{\prime} \neq j, i=1,2 \cdots, K\right\}$, the expected time becomes $T_{V_{i}^{\prime}, j}^{(K)}+1$. Thus, we have

$$
T_{V_{i}, j}^{(K)}=1+\sum_{j \notin V_{i}^{\prime}} P_{V_{i}, V_{i}^{\prime}} T_{V_{i}^{\prime}, j}^{(K)},
$$

where $P_{V_{i}, V_{i}^{\prime}}$ represents the transition probability from node set $V_{i}$ to $V_{i}^{\prime}$ for the $K$ searchers in one step. When considering all possible initial conditions (i.e., the starting position for the $K$ searchers), equation (1) can be rewritten in matrix form as

$$
T_{j}^{(K)}(1)=\mathbf{e}+\widetilde{\mathbf{P}} T_{j}^{(K)}(1),
$$

where $\mathbf{e}$ is a $\left(\begin{array}{c}N+K-2 \\ m\end{array}\right)$-dimensional vector with all entries 1 and $\widetilde{\mathbf{P}}$ is the submatrix of the aggregated transition probability matrix $\mathbf{P}=\left\{P_{V_{i}, V_{i}^{\prime}}\right\}$ by deleting the set of rows and columns with indexes $\left\{V_{i} \mid j \in V_{i}\right\}$. Since $(\mathbf{I}-\widetilde{\mathbf{P}})$ is a reversible matrix [28], we have

$$
T_{j}^{(K)}(1)=(\mathbf{I}-\widetilde{\mathbf{P}})^{-1} \mathbf{e},
$$

where $\mathbf{I}$ is an identity matrix. Repeatedly, suppose that we have already obtained the mean random cover time $T_{V_{i}, \Omega_{l}}^{(K)}(l)$ for $l=1,2, \cdots, m-1$. Consequently, we will consider 
how to derive the mean random cover time $T_{V_{i}, \Omega_{m}}^{(K)}(m)$ exactly from the known information. Similarly, it is easy to verify that the equation $T_{V_{i}, \Omega_{m}}^{(K)}(m)=T_{V_{i},\left(\Omega_{m} \backslash \Omega_{r}\right)}^{(K)}(m-r)$ holds for $\Omega_{r} \subset V_{i}$. Since $V_{i} \bigcap \Omega_{m}=\emptyset$, we have

$$
T_{V_{i}, \Omega_{m}}^{(K)}(m)=\sum_{r=1}^{m-1} \sum_{\Omega_{r} \subset V_{i}} P_{V_{i}, \Omega_{r}}\left(T_{V_{i},\left(\Omega_{m} \backslash \Omega_{r}\right)}^{(K)}(m-r)+1\right)+\sum_{V_{l} \cap \Omega_{m}=\emptyset} P_{V_{i}, V_{l}}\left(T_{V_{l}, \Omega_{m}}^{(K)}(m)+1\right) .
$$

We can rewrite the Eq. (4) in matrix form as

$$
T_{\Omega_{m}}^{(K)}=\overline{\mathbf{e}}+\sum_{r=1}^{m-1} \sum_{\Omega_{r} \subset V_{i}} \bar{P}_{\Omega_{r}} \times T_{V_{i},\left(\Omega_{m} \backslash \Omega_{r}\right)}^{(K)}(m-r) \overline{\mathbf{e}}+\overline{\mathbf{P}} T_{\Omega_{m}}^{(K)}(m),
$$

where $T_{\Omega_{m}}^{(K)}(m)$ is an $(N-m)$-dimensional vector; $\overline{\mathbf{e}}$ is the all-ones vector; $\overline{\mathbf{P}}$ is the submatrix of the aggregated transition probability matrix $\mathbf{P}$ by deleting the set of rows and columns with indexes $V_{i} \bigcap \Omega_{m} \neq \emptyset ; \bar{P}_{\Omega_{r}}$ represents the column indexes $V_{i} \bigcap \Omega_{r} \neq \emptyset$. Since the matrix $(\mathbf{I}-\overline{\mathbf{P}})$ is reversible [28], Eq. (5) can be recast as

$$
T_{\Omega_{m}}^{(K)}(m)=(\mathbf{I}-\overline{\mathbf{P}})^{-1}\left(\overline{\mathbf{e}}+\sum_{r=1}^{m-1} \sum_{\Omega_{r} \subset V_{i}} \bar{P}_{\Omega_{r}} \times T_{V_{i},\left(\Omega_{m} \backslash \Omega_{r}\right)}^{(K)}(m-r) \overline{\mathbf{e}}\right) .
$$

Clearly, equation (6) provides an iterative approach for calculating the MRCT analytically. Moreover, for quantifying the search efficiency at a global scale, we introduce the global $\operatorname{MRCT}\left\langle T^{(K)}\right\rangle(m)=\frac{1}{\left(\begin{array}{c}N \\ m\end{array}\right)\left(\begin{array}{c}N+K-1 \\ K\end{array}\right)} \sum_{\Omega_{m}} T_{\Omega_{m}}^{(K)}(m)$, by averaging Eq. (6) over all possible source and target sets. Interestingly, utilizing the annealed network approach [29] and the ShermanMorrison formula [30], we find the logarithmic growth pattern of $\left\langle T^{(K)}\right\rangle(m)$ versus $m$ (see Appendix A):

$$
\left\langle T^{(K)}\right\rangle(m) \approx \gamma \ln (m+1) / K
$$

where $\gamma$ is the growth rate highly related to the global mean first passage time (MFPT) $\left\langle T^{(1)}\right\rangle$, to which previous studies have been devoted $[5,6]$. Clearly, this expression unveils a universal dependence of the global $\operatorname{MRCT}\left\langle T^{(K)}\right\rangle(m)$ on the search efficiency of the individual searchers and on the number of searchers.

In order to validate the logarithmic growth pattern, we start from addressing a generic random walk of which the transition probability is $p_{i j}=a_{i j} / \sum_{j} a_{i j}$, where $a_{i j}$ is an element of the adjacency matrix [5]. We consider $K$ random searchers moving on the BarabásiAlbert (BA) network [31] and three real networks (i.e., the "Karate club" network [32], the "Chesapeake" network [33], and the "Football" network [34]). Figure 1 reveals a quantitative 
agreement between the numerical results and the theoretical predictions given by Eq. (7). Both the number of searchers dependence and the the number of targets dependence are unambiguously captured by our theoretical expression (i.e., Eq. (7)), as shown by the data collapse of the theoretical predictions. These results show that the global $\operatorname{MRCT}\left\langle T^{(K)}\right\rangle(m)$ grows logarithmically with the number of targets, as expected.
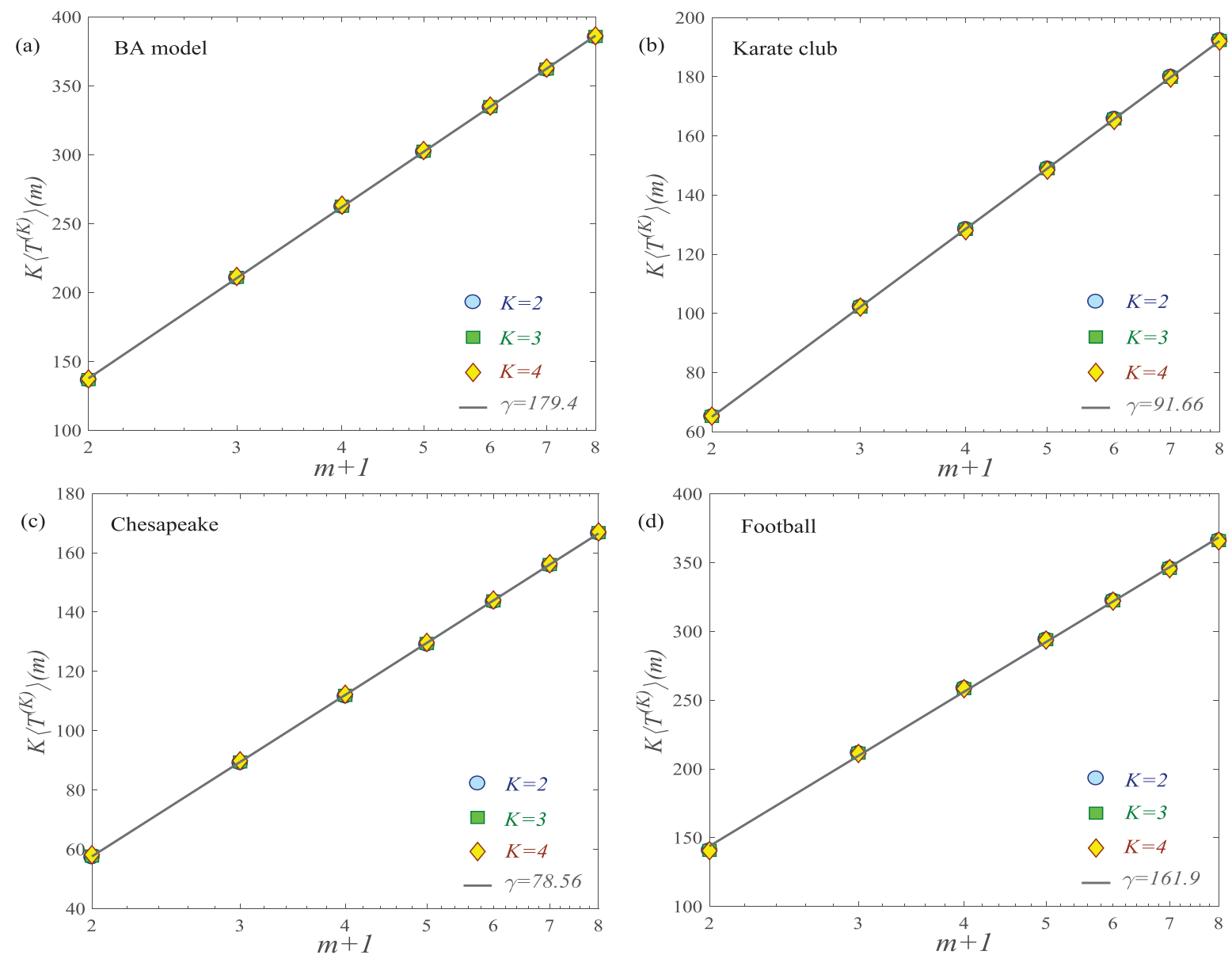

FIG. 1: The semilogarithmic plots show the quantity $K\left\langle T^{(K)}\right\rangle(m)$ as a function of the number of targets $m$ with respect to different number of searchers $K$ on (a) the BA model, (b) the "Karate club" network, (c) the "Chesapeake" network, and (d) the "Football" network. The values of $\gamma$ are obtained from the slopes of the fitting lines.

As a further validation, we address degree-biased random walks with the preferential transition probability $p_{i j}=\frac{a_{i j} k_{j}^{\alpha}}{\sum_{j} a_{i j} k_{j}^{\alpha}}$, where $\alpha$ is the tuning parameter and $k_{j}$ is the degree of node $j$ [35]. Clearly, the tuning parameter $\alpha$ determines the preference of visiting high or low degree node at each time step, which consequently influences search efficiency of the 
searcher. In Figs. 2(a) and 2(b), we explore the effect of the tuning parameter $\alpha$ on the global MRCT $\left\langle T^{(K)}\right\rangle(m, \alpha)$. It is shown that all profiles present the same tendency regarding different number of searchers $K$ and the number of targets $m$. In particular, the optimal tuning exponent $\alpha_{\text {opt }}$ of a biased random walk strategy, where the minimum $\left\langle T^{(K)}\right\rangle(m, \alpha)$ is achieved, is robust and is independent of the number of targets $m$ as well as the number of searchers $K$. We then confirm the validity of the logarithmic growth pattern by taking two representative biased random walk strategies — the optimal biased random walk (OBRW) and the maximal entropy random walk (MERW). For the MERW, the transition probability is $p_{i j}=\frac{a_{i j}}{\lambda} \frac{\mu_{j}}{\mu_{i}}$, where $\lambda$ is the largest eigenvalue of the adjacency matrix $\mathbf{A}$ and $\mu_{j}$ is the $j^{\text {th }}$ element of the corresponding principal eigenvector $\mu$ [25]. Clearly, the numerical results match the theoretical predictions, see Figs. 2(c)-2(f). These findings further confirm that the logarithmic growth pattern is a universal principle governing multiple targets search.

Moreover, we show that the logarithmic growth pattern is also appropriate for the persistent random walks (PRW). In contrast to the previous memoryless search strategies, the persistent random walk has one-step memory, i.e., the future motion of the random searcher depends not only on its current position, but also on its previous location information [26]. More specifically, at each time step, the random searcher has a probability $\frac{1+\epsilon}{2}$ to continue in the same direction as the previous step, while with the probability $\frac{1-\epsilon}{2}$ to go in the inverse direction. Here we consider the case of the PRW on a 1D lattice with the periodic boundary condition, as described in Fig. 3(a). To eliminating the effect of one-step memory, we built a directed network from the 1D lattice model in which node $i+(i-)$ means in the previous step, the searcher moves to node $i$ following a clockwise (anticlockwise) direction (see Fig. 3(b)). A directed edge from node $i+$ to $j$ - exists whenever the transition probability from node $i$ to node $j$ following a anticlockwise direction is larger than zeros. It is easy to see that the PRW moving on the constructed directed network satisfies the features of Markov process. Thus, we can apply our paradigm for calculating MRCT directly and observe how the search time increases with respect to the number of targets.

We first observe the size effect on the global MFPT $\left\langle T^{(1)}\right\rangle(\epsilon)$ of the PRW. Figure 3(c) shows that the profiles present the same decreasing tendency for different network sizes $N$. The way in which $\left\langle T^{(1)}\right\rangle(\epsilon)$ scales with network size $N$ seems to follow a power law behavior as shown in the inset of Fig. 3(c). Interestingly, we find that our theoretical predictions fit well with the analytical results given by Eq. (6). The results further demonstrate that the 

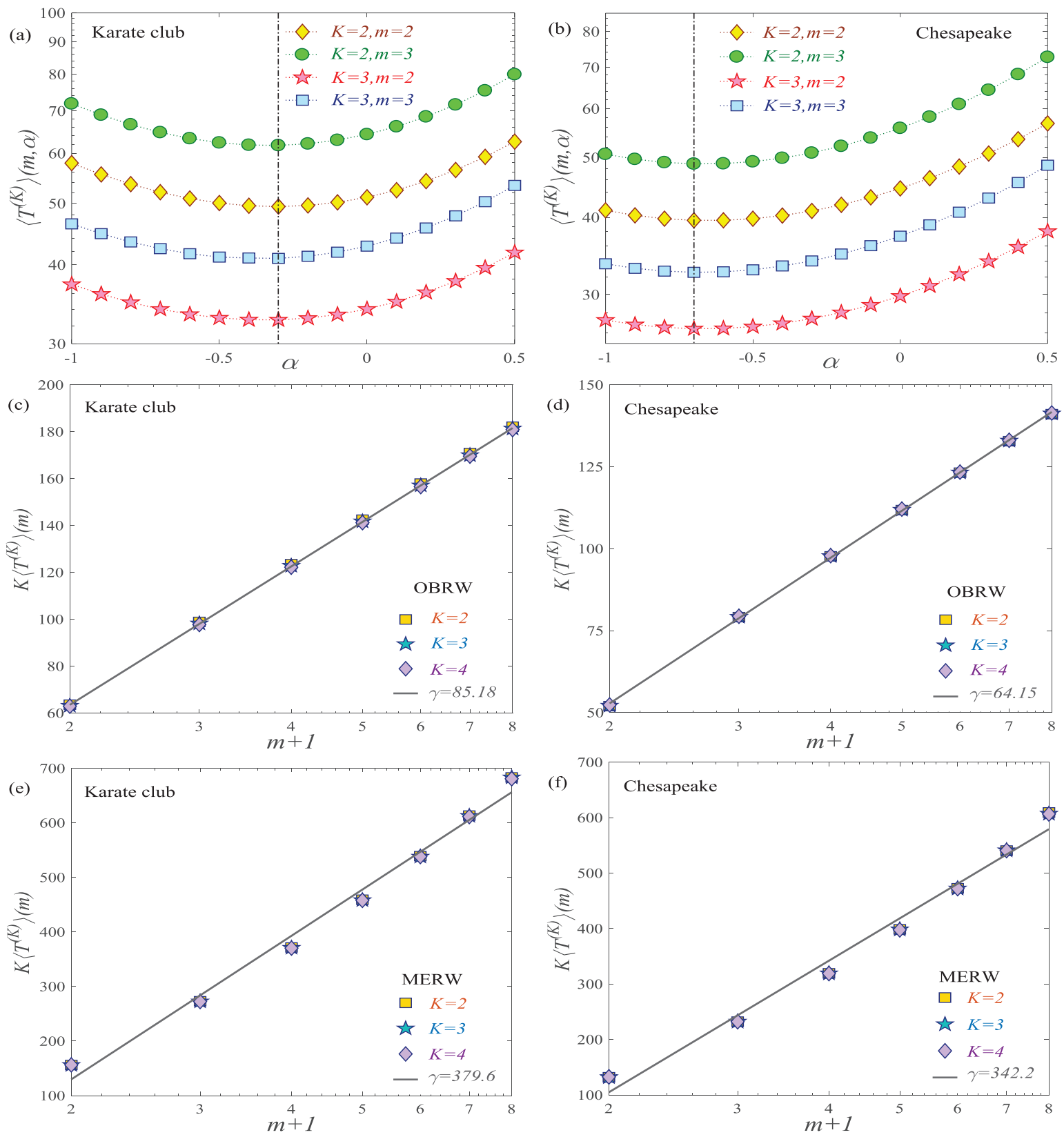

FIG. 2: The global MRCT $\left\langle T^{(K)}\right\rangle(m, \alpha)$ as a function of the tuning parameter $\alpha$ on (a) the "Karate club" network and (b) the "Chesapeake" network. The vertical dashed lines indicate the positions of $\alpha_{\text {opt }}$ for each network. The semilogarithmic plots show the quantity $K\left\langle T^{(K)}\right\rangle(m)$ as a function of the number of targets for performing the optimal biased random walks on (c) the "Karate club" network and (d) the "Chesapeake" network, while the corresponding results of adopting the maximal entropy random walks are shown in (e) and (f). The values of $\gamma$ are achieved from the slopes of the fitting straight lines. 
logarithmic growth pattern is also suitable for non-Markov random search process. Note that here we choose the parameter $\epsilon=0.5$ for convenience. In fact, the logarithmic growth pattern is a general principle for an arbitrary parameter $\epsilon$.
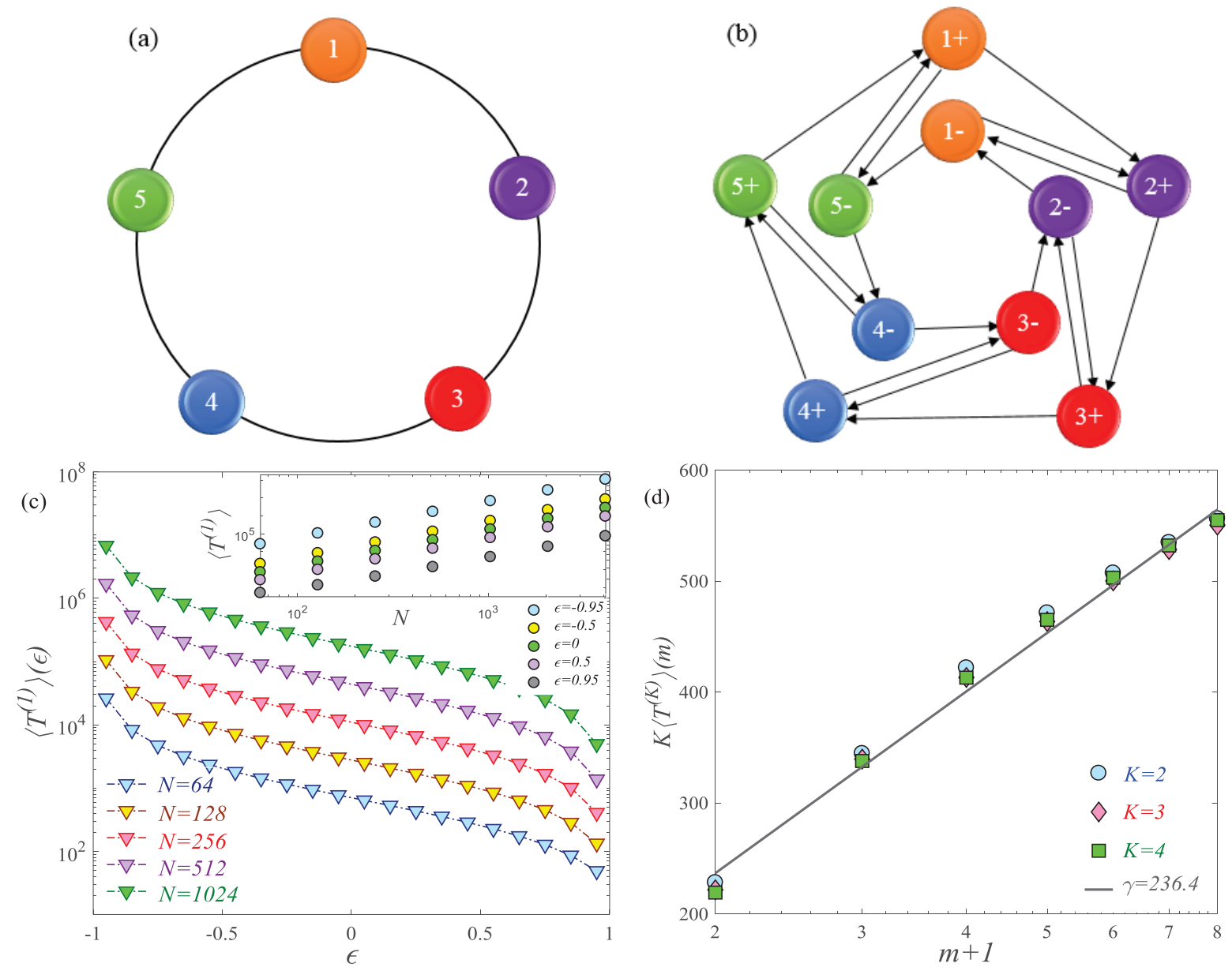

FIG. 3: (a) Schematic illustration of a 1D lattice with the periodic boundary condition. (b) The directed network constructed from the $1 \mathrm{D}$ lattice model. (c) The global MFPT $\left\langle T^{(1)}\right\rangle(\epsilon)$ as a function of $\epsilon$ for the PRW on 1D lattice over different network sizes $N$. In the inset, we show the behaviors of $\left\langle T^{(1)}\right\rangle$ versus $N$ with respect to parameter $\epsilon$. (d) The semilogarithmic plot shows the quantity $K\left\langle T^{(K)}\right\rangle(m)$ as a function of the number of targets.

Furthermore, we address a special anomalous random walk — the intermittent random walk (IRW) for which the searcher can select two distinct transition patterns at each time step [27]. In particular, with probability $\mu$, the searcher jumps according to the unbiased random walk rule, while with the probability $1-\mu$, the searcher leaves the lattice and lands at a site $L$ distance away from the departure site. Clearly, the damping factor $\mu$ plays an 
important role in shaping the behavior of the IRW. To explore this effect, we study the global MFPT $\left\langle T^{(1)}\right\rangle(\mu)$ of the IRW on a 1D lattice. The result presented in Fig. 4(a) clearly shows the presence of a minimum $\left\langle T^{(1)}\right\rangle$ for different network sizes $N$. However, it is easy to see that the minimum position changes gradually with increasing network size N (see in the inset of Fig. 4(a)). Such behavior is clearly distinct from that of the PRW, where the optimal tuning exponent remains stable irrespective of network size. Nonetheless, our theoretical prediction of Eq. (7) can still capture multiple target search based on the IRW, as shown by all data collapse of the theoretical predictions in Fig. 4(b). The result provides a further validation of our finding that the logarithmic growth pattern is a universal principle controlling multiple target search.
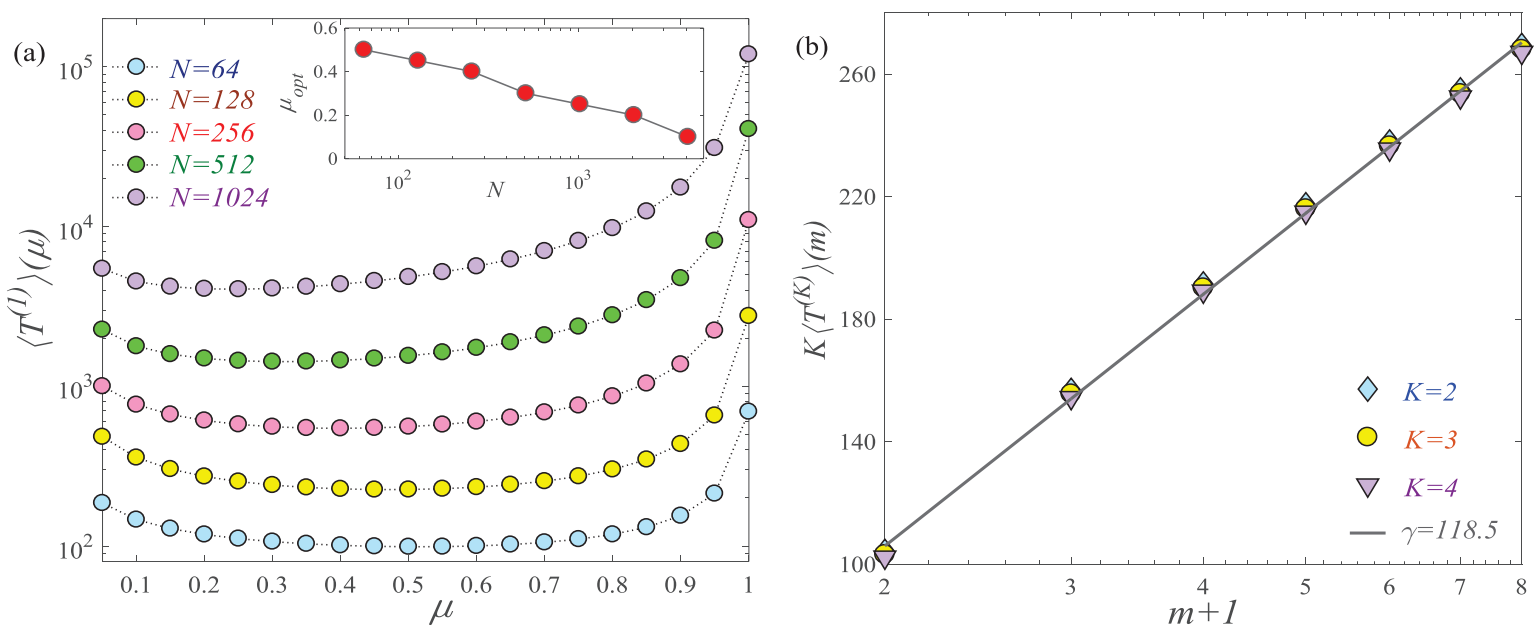

FIG. 4: (a) The global MFPT $\left\langle T^{(1)}\right\rangle(\mu)$ as a function of $\mu$ for the IRW on 1D lattice over different network sizes $N$. In the inset, we show the plot of $\mu_{\text {opt }}$ vs $N$ for $L=10$. (b) The semilogarithmic plot exhibits the quantity $K\left\langle T^{(K)}\right\rangle(m)$ as a function of the number of targets.

Finally, in practice, we sometimes find that the properties of individual random searchers are distinct. For example, a prey species usually have several predators for which different predators adopt distinct search strategies. A fascinating problem is how search efficiency of multiple searchers related to that of the individual searchers. To address this issue, we consider $K$ distinct searchers moving on the network independently and concurrently. We assume that they do not interact with each other in the whole search process. Utilizing the annealed network approach [29] and the Sherman-Morrison formula [30], we find the harmonic law exploring the contribution and effect of each individual searcher (see Appendix 
B)

$$
\left\langle T^{(K)}\right\rangle(m) \approx \frac{1}{\sum_{l=1}^{K} \frac{1}{\left\langle T_{l}^{(1)}\right\rangle(m)}},
$$

where $\left\langle T_{l}^{(1)}\right\rangle(m)$ is the global MRCT of the $l^{\text {th }}$ searcher for finding $m$ targets given in advance. In particular, when transition properties of the searchers are identical, it is easy to verify that the harmonic law of Eq. (8) degenerates to that of Eq. (7), as expected. Strikingly, the result of Eq. (8) unveils the contributions and effects of the individual searchers on multi-target search. To demonstrate the harmonic law, we investigate the global $\operatorname{MRCT}\left\langle T^{(K)}\right\rangle(m)$ of multiple distinct searchers adopting the previous analyzed search strategies. It is shown that the analytic results agree well with the theoretical predictions given by Eq. (8), as illustrated in Fig. 5. The results indicate that the harmonic law of Eq. (8) unambiguously captures the search time of multiple distinct searchers, which generalizes the previous results of a single target search as reported in Ref. [36]. These findings point out that the search time of multiple random searchers are controlled by the combination of the logarithmic growth pattern and the harmonic law together.

In summary, we have presented a fundamental framework for characterizing and quantifying multiple random searchers on networks. We propose an iterative approach to calculate the general expression of mean random cover time analytically. Remarkably, we find two fundamental principles (i.e., the logarithmic growth pattern and the harmonic law) controlling the search time of multiple random searchers. In particular, the logarithmic growth pattern describes the effect of increasing the number of targets, while the harmonic law explores how the search time of multiple random searchers is related to that of each individual searcher in the same environment. These two universal principles are demonstrated in examples drawn from degree-biased random walks, maximal entropy random walks, persistent random walks, and intermittent search strategies. Our findings will deepen the understanding of various diffusion processes on networks and will also facilitate us to realize diverse dynamical processes on networks including epidemic spreading [37], synchronization [38, 39], and transportation [40].

This work provides a general framework for studying random search processes, which incorporates our previous works (i.e., the multitarget search [22] and the multiple random searchers [36]) as two special cases. Moreover, here we show that the logarithmic growth pattern and the harmonic law are also established on persistent random walks and intermit- 

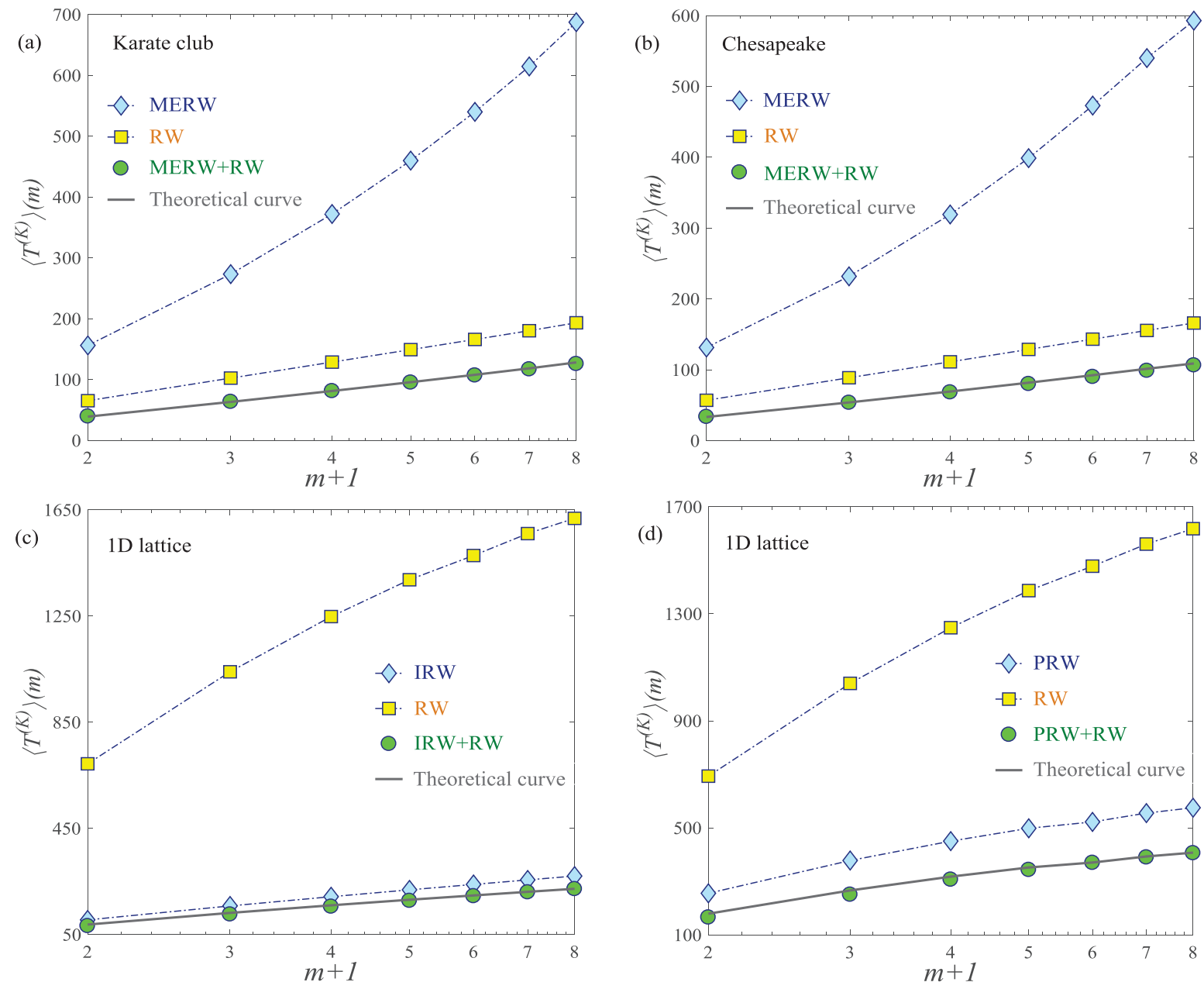

FIG. 5: The global MRCT $\left\langle T^{(K)}\right\rangle(m)$ of multiple distinct random searchers adopting the previous analyzed search strategies. The solid lines are the theoretical predictions given by $c / \sum_{l=1}^{K} \frac{1}{\left\langle T_{l}^{(1)}\right\rangle(m)}$ on (a) $c=0.86$, (b) $c=0.87$, (c) $c=0.98$, and (d) $c=0.99$.

tent search strategies, which further extends our previous findings. Meanwhile, in the case of persistent random walks, we propose constructing a directed network for solving the effect of one-step memory. This idea may provide a new path for calculating mean first passage time of non-Markovian random search analytically. Moreover, for convenience, we assume that multiple random searchers move independently on networks without information sharing. The investigation of multiple random searchers with interaction calls for additional research effects. 


\section{APPENDIXES}

\section{A. The origin of the logarithmic growth pattern of multiple identical random} searchers

We study $K$ identical random searchers traversing on a connected network independently and concurrently. At each time step, the individual searcher jumps to node $j$ from current node $i$ with a transition probability $f\left(x_{j}\right)$ depending on the node property $x_{j}$ (degree, betweenness, clustering coefficient, etc.). Take biased random walks as an example, the transition probability $f\left(x_{j}\right)$ is defined as $f\left(x_{j}\right)=k_{j}^{\alpha}$, where $\alpha$ is the tuning parameter and $k_{j}$ is the degree of node $j$. For an uncorrelated network, we can reinterpret its adjacency matrix A as a weighted fully connected graph $\widetilde{\mathbf{A}}$ based on the annealed network approach [29]. Specifically, the entry $\widetilde{a}_{i j}=\frac{k_{i} k_{j}}{N\langle k\rangle}$ defines the connection probability between nodes $i$ and $j$, where $\langle k\rangle$ represents the average degree of the whole network. In this context, the transition probability of the searcher becomes $p_{i j}=\frac{k_{j} f\left(x_{j}\right)}{\sum_{j} k_{j} f\left(x_{j}\right)}$. Likewise, the aggregated transition matrix $\mathbf{P}$ can be expressed as

$$
\mathbf{P}=\frac{1}{\left(\sum_{i=1}^{N} k_{i} f\left(x_{i}\right)\right)^{K}}\left(\begin{array}{cccccc}
\left(k_{1} f\left(x_{1}\right)\right)^{K} & K k_{1} f\left(x_{1}\right)\left(k_{2} f\left(x_{2}\right)\right)^{K-1} & K k_{1} f\left(x_{1}\right)\left(k_{3} f\left(x_{3}\right)\right)^{K-1} & \cdots & K k_{N-1} f\left(x_{N-1}\right)\left(k_{N} f\left(x_{N}\right)\right)^{K-1} & \left(k_{N} f\left(x_{N}\right)\right)^{K} \\
\left(k_{1} f\left(x_{1}\right)\right)^{K} & K k_{1} f\left(x_{1}\right)\left(k_{2} f\left(x_{2}\right)\right)^{K-1} & K k_{1} f\left(x_{1}\right)\left(k_{3} f\left(x_{3}\right)\right)^{K-1} & \cdots & K k_{N-1} f\left(x_{N-1}\right)\left(k_{N} f\left(x_{N}\right)\right)^{K-1} & \left(k_{N} f\left(x_{N}\right)\right)^{K} \\
\vdots & \vdots & \vdots & \vdots & \vdots & \vdots \\
\left(k_{1} f\left(x_{1}\right)\right)^{K} & K k_{1} f\left(x_{1}\right)\left(k_{2} f\left(x_{2}\right)\right)^{K-1} & K k_{1} f\left(x_{1}\right)\left(k_{3} f\left(x_{3}\right)\right)^{K-1} & \cdots & K k_{N-1} f\left(x_{N-1}\right)\left(k_{N} f\left(x_{N}\right)\right)^{K-1} & \left(k_{N} f\left(x_{N}\right)\right)^{K}
\end{array}\right) .
$$

Utilizing the Sherman-Morrison formula [30], the inverse of the matrix $(\mathbf{I}-\overline{\mathbf{P}})$ in Eq. (6) becomes

$$
(\mathbf{I}-\overline{\mathbf{P}})^{-1}=\mathbf{I}+\frac{\left(\sum_{i=1}^{N} k_{i} f\left(x_{i}\right)\right)^{K}}{\left(\sum_{i=1}^{m} k_{i} f\left(x_{i}\right)\right)^{K}-\left(\sum_{i=1}^{m} k_{i} f\left(x_{i}\right)-\sum_{l \in \Omega_{m}} k_{l} f\left(x_{l}\right)\right)^{K}} \overline{\mathbf{P}} .
$$

Inserting Eq. (10) into Eq. (6) with a few simple algebraic manipulations, we have a recursion relation

$$
\left\langle T^{(K)}\right\rangle(m) \approx \frac{\sum_{i=1}^{N} k_{i} f\left(x_{i}\right)}{N K m} \sum_{i=1}^{N} \frac{1}{k_{i} f\left(x_{i}\right)}+\left\langle T^{(K)}\right\rangle(m-1) .
$$

Using the lower bound $\ln (m+1)$ for estimating the partial sums of the harmonic series $\sum_{i}^{m} 1 / i$, Eq. (11) can be solved to obtain

$$
\left\langle T^{(K)}\right\rangle(m) \approx \gamma \ln (m+1) / K
$$

where $\gamma$ represents the growth rate highly related to the global mean first passage time $\left\langle T^{(1)}\right\rangle$. Our result unveils that the global mean random cover time follows the logarithmic 
growth pattern with respect to the number of targets.

\section{B. The origin of the harmonic law in controlling multiple distinct random} searchers

We now consider a more complex case, where $K$ random searchers adopt distinct random search strategies. Specifically, at each time step, the $l^{\text {th }}$ searcher moves from node $i$ to node $j$ with a transition probability $f_{l}\left(x_{j}\right)$. Utilizing the annealed network approach to reinterpret the network as a fully connected graph [29], we have the associated aggregated transition probability $\mathbf{P}$

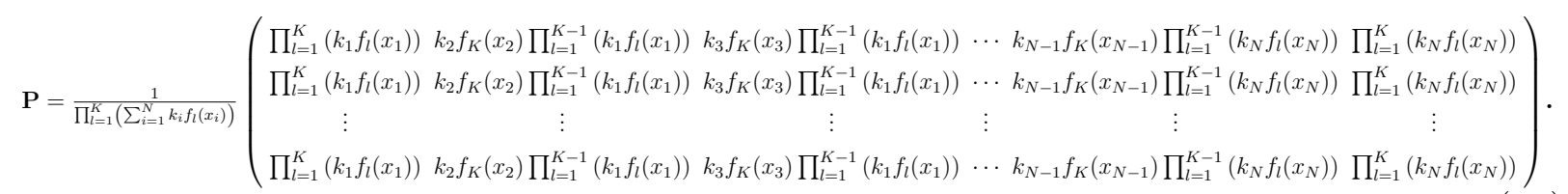

Applying the Sherman-Morrison formula [30], the inverse of the matrix $(\mathbf{I}-\overline{\mathbf{P}})$ in Eq. (6) becomes

$$
(\mathbf{I}-\overline{\mathbf{P}})^{-1}=\mathbf{I}+\frac{\prod_{l=1}^{K}\left(\sum_{i=1}^{N} k_{i} f_{l}\left(x_{i}\right)\right)}{\prod_{l=1}^{K}\left(\sum_{i=1}^{N} k_{i} f_{l}\left(x_{i}\right)\right)-\prod_{l=1}^{K}\left(\sum_{i=1}^{N} k_{i} f_{l}\left(x_{i}\right)-\sum_{j \in \Omega_{m}} k_{j} f_{l}\left(x_{j}\right)\right)} \overline{\mathbf{P}} .
$$

Substituting into Eq. (6) with some calculations, we have

$$
\left\langle T^{(K)}\right\rangle(m) \approx \frac{1}{N m} \sum_{j=1}^{N}\left(\frac{1}{\sum_{l=1}^{K} \frac{k_{j} f_{l}\left(x_{j}\right)}{\sum_{i=1}^{N} k_{i} f_{l}\left(x_{i}\right)}}\right)+\left\langle T^{(K)}\right\rangle(m-1) .
$$

Meanwhile, the global MRCT for the $l^{\text {th }}$ searcher can be approximated by $\left\langle T_{l}^{(1)}\right\rangle(m) \approx$ $\frac{\sum_{i=1}^{N} k_{i} f_{l}\left(x_{i}\right)}{N} \sum_{i=1}^{N} \frac{1}{k_{i} f_{l}\left(x_{i}\right)} \ln (m+1)$. Thus, we obtain

$$
\left\langle T^{(K)}\right\rangle(m) \approx \frac{1}{\sum_{l=1}^{K} \frac{1}{\left\langle T_{l}^{(1)}\right\rangle(m)}} .
$$

Clearly, equation (16) reveals that the global MRCT of multiple random searchers follows the harmonic law with respect to the global MRCTs of the individual searchers.

This research has been supported, in part, by General Research Fund 26211515 from the Research Grants Council of Hong Kong, and Innovation and Technology Fund ITS/369/14FP from the Hong Kong Innovation and Technology Commission. J.Z. is supported 
by National Science Foundation of China NSFC 61573107. MS is supported by Australian Research Council Discovery Project DP 140100203.

[1] N. Dongari, Y. H. Zhang, and J. M. Reese, J. Phys. D Appl. Phys. 44, 125502 (2011).

[2] G. M. Viswanathan, M. G. E. da Luz, E. P. Raposo, and H. E. Stanley, The Physics of Foraging: An Introduction to Random Searches and Biological Encounters (Cambridge University Press, 2011).

[3] T. Perkins, E. Foxall, L. Glass, and R. Edwards, Nat. Commun. 5, 5121 (2014).

[4] A. L. Lloyd and R. M. May, Science 292, 1316 (2001).

[5] J. D. Noh and H. Rieger, Phys. Rev. Lett. 92, 118701 (2004).

[6] N. Perra, A. Baronchelli, D. Mocanu, B. Goncalves, R. Pastor-Satorras, and A. Vespignani, Phys. Rev. Lett. 109, 238701 (2012).

[7] T. F. Weng, M. Small, J. Zhang, and P. Hui, Sci. Rep. 5, 17309 (2015).

[8] T. F. Weng, J. Zhang, K. Moein, M. Small, Z. Rui, and P. Hui, Sci. Rep. 6, 37547 (2016).

[9] M. L. Heuzé et al., Immunol. Rev. 256, 240-254 (2013).

[10] G. M. Viswanathan, E. P. Raposo, and M. G. E. da Luz, Phys. Life Rev. 5, 133 (2008).

[11] M. Chupeau, O. Bénichou, and R. Voituriez, Nat. Phys. 11, 844 (2015).

[12] G. M. Viswanathan, S. V. Buldyrev, S. Havlin, M. G. E. da Luz, E. P. Raposo, and H. E. Stanley, Nature (London) 401, 911 (1999).

[13] F. Bartumeus, J. Catalan, U. L. Fulco, M. L. Lyra, and G. M. Viswanathan, Phys. Rev. Lett. 88, 097901 (2002).

[14] M. C. Santos, G. M. Viswanathan, E. P. Raposo, and M. G. E. da Luz, Phys. Rev. E 72, $046143(2005)$.

[15] C. L. Faustino, M. L. Lyra, E. P. Raposo, G. M. Viswanathan, and M. G. E. da Luz, Europhys. Lett. 97, 50005 (2012).

[16] M. E. Wosniack, E. P. Raposo, G. M. Viswanathan, and M. G. E. da Luz, Phys. Rev. E 92, $062135(2015)$.

[17] D. J. Aldous, SIAM J. Discrete Math. 3, 450 (1990).

[18] I. Adler, S. Oren, and S. M. Ross, J. Appl. Prob. 40, 513 (2003).

[19] K. R. Coutinho, M. D. Coutinho-Filho, M. A. F. Gomes, and A. M. Nemirovsky, Phys. Rev. 
Lett. 72, 3745 (1994).

[20] M. S. Nascimento, M. D. Coutinho-Filho, and C. S. O. Yokoi, Phys. Rev. E 63, 066125 (2001).

[21] J. R. G. Mendonca, Phys. Rev. E 84, 022103 (2011).

[22] T. F. Weng, J. Zhang, M. Small, J. Yang, F. H. Bijarbooneh, and P. Hui, Chaos 27, 093103 (2017).

[23] N. Alon, C. Avin, M. Koucký, G. Kozma, Z. Lotker, and M. R. Tuttle, Combin. Probab. Comput. 20, 481 (2011).

[24] C. Cooper, A. Frieze, and T. Radzik, SIAM J. Discrete Math. 23, 1738 (2009).

[25] Y. Lin and Z. Z. Zhang, Sci. Rep. 4, 5365 (2014).

[26] Z. Sadjadi and M. F. Miri, Phys. Rev. E 78, 061114 (2008).

[27] G. Oshanin, K. Lindenberg, H. Wio, and S. Burlatsky, J. Phys. A: Math. Theor. 42, 434008 (2009).

[28] C. M. Grinstead and J. L. Snell, Introduction to Probability (American Mathematical Society, 2006).

[29] S. N. Dorogovtsev, A. V. Goltsev, and J. F. F. Mendes, Rev. Mod. Phys. 80, 1275 (2008).

[30] J. Sherman and W. J. Morrison, Ann. Math. Stat. 21, 124 (1950).

[31] A. Barabási and R. Albert, Science 286, 509 (1999).

[32] W. W. Zachary, J. Anthropol. Res. 33, 452 (1977).

[33] D. Baird and R. E. Ulanowicz, Ecol. Monogr. 59, 329 (1989).

[34] M. Girvan and M. E. J. Newman, Proc. Natl. Acad. Sci. USA 99, 7821 (2002).

[35] A. Fronczak and P. Fronczak, Phys. Rev. E 80, 016107 (2009).

[36] T. F. Weng, J. Zhang, M. Small, and P. Hui, Phys. Rev. E 95, 052103 (2017).

[37] I. Pozzana, K. Y. Sun, and N. Perra, Phys. Rev. E 96, 042310 (2017).

[38] C. G. Gu, M. Tang, and H. J. Yang, Sci. Rep. 6, 28878 (2016).

[39] C. G. Gu and H. J. Yang, Chaos 27, 093108 (2017).

[40] C. Nicolaides, L. Cueto-Felgueroso, and R. Juanes, Phys. Rev. E 82, 055101(R) (2010). 